Check for updates

Cite this: RSC Adv., 2017, 7, 47261

Received 30th June 2017

Accepted 29th September 2017

DOI: 10.1039/c7ra07227a

rsc.li/rsc-advances

\section{Synthesis of an oligomer ruthenium complex and its catalysis in the oxidation of alcohols $\uparrow$}

\author{
Yuecheng Zhang, Wenge Huo, Hong-Yu Zhang and Jiquan Zhao (DD * \\ A ligand with both a terpyridine and a pyridine-2,6-dicarboxylate group (abbreviated as terpy-pydic) was \\ designed and synthesized. This ligand reacted with $\left[\mathrm{Ru}(p \text {-cymene }) \mathrm{Cl}_{2}\right]_{2}$ to afford a novel oligomer \\ ruthenium complex named as oligomer-Ru(terpy)(pydic) which was characterized thoroughly. Under the \\ catalysis of this oligomer ruthenium complex, different sorts of secondary alcohols were oxidized to the \\ corresponding kenones by the oxidant tert-butyl hydroperoxide. Besides, this catalyst can be readily \\ recovered and recycled several times without a large loss of its efficiency.
}

\section{Introduction}

Oxidation of alcohols to their corresponding carbonyl compounds is a fundamental transformation in the synthesis of various valuable chemicals such as fragrances, pharmaceuticals, dyes and food additives. ${ }^{1-4}$ Traditionally, this transformation is realized with a stoichiometric or even an over-stoichiometric amount of $\mathrm{MnO}_{2},{ }^{5}$ chromium(vI) oxide, ${ }^{1,6-8}$ and hypervalent iodine compounds ${ }^{9-13}$ as oxidants, which will bring about serious environmental problems. To overcome these problems, many efforts have been made to explore transition-metal-catalyzed processes using environmentally benign oxidants, especially molecular oxygen as the oxidant. ${ }^{14-27}$

Due to the existence of redox ion pairs $\mathrm{Ru}^{n+} / \mathrm{Ru}^{(n+2)}$, ruthenium complexes have emerged as efficient catalysts for the oxidation of various substrates. ${ }^{28-42}$ Among all of them, some $N, N, N$-tridentate ligand ruthenium complexes exhibited good performances in the oxidation of alcohols in view of their high efficiency and selectivity. ${ }^{24-26,36}$ For instances, both Ru(terpyridine)(pydic) and its analogue $\mathrm{Ru}(\mathrm{bbp})($ pydic) gave good to excellent yields of ketones in the oxidation of various secondary alcohols with hydrogen peroxide as oxidant. ${ }^{24,25}$ Another similar ruthenium complex $\mathrm{Ru}($ pymieb)(pydic) reported by us also exhibited high efficiency in the oxidation of secondary alcohols to ketones with TBHP as oxidant under mild conditions. ${ }^{26}$ Despite of their excellent performances in the reaction, it is difficult to separate the expensive homogeneous catalysts with products, which leads to impossible recycling in application. Heterogenization of the ruthenium complexes has been expected to resolve this problem. To this end, some ruthenium complexes were supported on organic or inorganic supports and the immobilized complexes

School of Chemical Engineering and Technology, Hebei University of Technology, Tianjin 300130, PR China. E-mail: zhaojq@hebut.edu.cn; Fax: +86 22 60202926; Tel: +8622 60202926; +862260204279

$\dagger$ Electronic supplementary information (ESI) available. See DOI: $10.1039 / \mathrm{c} 7 \mathrm{ra0} 07227 \mathrm{a}$ exhibited good recyclability in the oxidation of various alcohols to the corresponding ketones. ${ }^{27,40-43}$ Although promising results were obtained, the immobilization processes were tedious in some cases, and the immobilization of the complex generally led to decrease of its activity due to mass transfer resistance of the reactants in the matrix of the support. Very recently, we used a polymer with a tridentate 2,6-bis(benzimidazol-2-yl)pyridine (bbp) unit in combination with disodium pyridine-2,6dicarboxylate (pydic) coordinating with $\mathrm{RuCl}_{3}$ to form a polymer-ruthenium complex poly-Ru(bbp)(pydic). ${ }^{44}$ This polymerruthenium complex showed high activity in the oxidation of various secondary alcohols to their corresponding ketones in high yields with TBHP as oxidant, and could be recycled for several times. Inspired by the catalytic results and center structures of the above catalysts, we designed and synthesized a ligand with both terpyridine and pydic moieties. This ligand coordinated with $\mathrm{Ru}^{2+}$ to afford an organo-ruthenium oligomer which showed good performances in the oxidation of alcohols with TBHP as oxidant in view of both activity and recyclability.

\section{Experimental}

\section{Materials}

Dimethyl 4-hydroxypyridine-2,6-dicarboxylate (1) was synthesized as reported method. ${ }^{45-47} 4^{\prime}$-(4-Bromomethylphenyl)$\left[2,2^{\prime}: 6^{\prime}, 2^{\prime \prime}\right]$ terpyridine (2) prepared as described in literature. ${ }^{48,49}$ tert-Butyl hydroperoxide (TBHP, $70 \%$ aqueous solution) was supplied by Energy Chemical. Dichloro( $p$-cymene)ruthenium(II) dimer $\left[\mathrm{Ru}(p \text {-cymene }) \mathrm{Cl}_{2}\right]_{2}$ was provided by J\&K Scientific Ltd. All the substrate alcohols were purchased from Alfa Aesar China Co. Ltd. All reagents were used as received without further purification.

\section{Characterization}

${ }^{1} \mathrm{H}$ NMR and ${ }^{13} \mathrm{C}$ NMR spectra were recorded on a Bruker AC-P 400 type spectrometer (400 MHz for ${ }^{1} \mathrm{H}$ and $101 \mathrm{MHz}$ for ${ }^{13} \mathrm{C}$ ). 
Chemical shifts $(\delta)$ were measured in ppm relative to TMS $\delta=$ 0 for ${ }^{1} \mathrm{H}$, or to $\mathrm{CDCl}_{3} \delta=77.2$ or DMSO $\delta=39.6$ for ${ }^{13} \mathrm{C}$ as internal standard. Mass spectra were measured using miorOTOF-QII mass spectrometer. Elemental analyses were carried out using Perkin-Elmer 2400 CHN analyzer. FT-IR spectra were recorded with a Bruker Vector 22 spectrophotometer with $\mathrm{KBr}$ pellets. Raman spectra were measured at room temperature using an inVia Reflex visible Raman spectrometer in backscattering geometry equipped with excitation argon ion lasers working at $532 \mathrm{~nm}$. UV-vis DRS were recorded with a Varian Cary 300 UV-visible spectrophotometer. Powder X-ray diffraction (XRD) patterns were performed on a Bruker AXS GMBH D8 Discover X-ray diffractometer using with a $\mathrm{Cu} \mathrm{K \alpha}$ radiation at $40 \mathrm{kV}$ and $40 \mathrm{~mA}\left(2 \theta=10-90^{\circ}\right)$. X-ray photoelectron spectroscopy (XPS) data were obtained on a PHI 1600 spectroscopy using $\mathrm{Al} \mathrm{K} \alpha$ radiation, using the carbon $\mathrm{C} 1 \mathrm{~s}$ peak at $284.6 \mathrm{eV}$ as a reference for the charge correction. SEM images and EDX spectra were taken with a Nova Nano SEM450 instrument and an Octane Pro Det. Transmission electron microscopy studies were carried out using a JEM-2100F Transmission Electron Microscope (TEM) at an accelerating voltage of $200 \mathrm{kV}$. The ruthenium content of the samples was determined using a Perkin Elmer Opfima $7300 \mathrm{~V}$ type ICP-AES instrument. Thermogravimetric analysis (TG-DTA) was evaluated using a TG/DTA 6300 thermogravimetric analyzer from 30 to $800{ }^{\circ} \mathrm{C}$ with a temperature rate of $10^{\circ} \mathrm{C} \mathrm{min}^{-1}$ in air. Oxidation reaction progress was monitored by a Gas Chromatograph (Shandong Lunan Ruihong SP-7800A) equipped with a SE 54 column of $30 \mathrm{~m}$.

\section{Catalyst preparation}

Synthesis of dimethyl $4-\left(4-\left(2,2^{\prime}: 6^{\prime}, 2^{\prime \prime}\right.\right.$-terpyridin-4'-yl) benzyloxy)pyridine-2,6-dicarboxylate (3). Into a solution of 2 $(1.87 \mathrm{~g}, 4.66 \mathrm{mmol})$ in dry DMF $(50 \mathrm{~mL})$ was added $\mathrm{K}_{2} \mathrm{CO}_{3}$ $(2.49 \mathrm{~g}, 18.0 \mathrm{mmol})$ and $1(0.95 \mathrm{~g}, 4.5 \mathrm{mmol})$ successively. The mixture was stirred at $80{ }^{\circ} \mathrm{C}$ for $14 \mathrm{~h}$. Then the reaction was stopped by adding water $(200 \mathrm{~mL})$, and the precipitate was collected by filtration. The precipitate was extracted with dichloromethane. The dichloromethane was removed by rotary evaporation to obtain $3(1.5 \mathrm{~g}, 63 \%)$ as a white solid. ${ }^{1} \mathrm{H}$ NMR (400 MHz, DMSO-d $\left.{ }_{6}\right): \delta 3.92(\mathrm{~s}, 6 \mathrm{H}), 5.51(\mathrm{~s}, 2 \mathrm{H}), 7.53-7.56(\mathrm{~m}$, $2 \mathrm{H}), 7.72(\mathrm{~d}, J=8.1 \mathrm{~Hz}, 2 \mathrm{H}), 7.89(\mathrm{~s}, 2 \mathrm{H}), 8.00-8.07(\mathrm{~m}, 4 \mathrm{H}), 8.69$ $(\mathrm{d}, J=8.0 \mathrm{~Hz}, 2 \mathrm{H}), 8.75(\mathrm{~s}, 2 \mathrm{H}), 8.78(\mathrm{~d}, J=4.0 \mathrm{~Hz}, 2 \mathrm{H}) .{ }^{13} \mathrm{C}$ NMR $\left(101 \mathrm{MHz}, \mathrm{CDCl}_{3}\right) \delta 53.3,70.4,114.9,118.9,121.4,123.9,127.9$, 128.2 , 135.5, 136.9, 139.0, 149.2, 149.5, 149.9, 156.0, 156.1, 165.1, 166.7 .

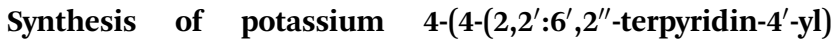
benzyloxy)pyridine-2,6-dicarboxylate (4). Compound 3 (3.40 g, $6.39 \mathrm{mmol})$ was added to a solution of $\mathrm{KOH}(8.2 \mathrm{~g}, 0.15 \mathrm{~mol})$ in THF $(150 \mathrm{~mL})$ and water $(200 \mathrm{~mL})$. The mixture was stirred at $80^{\circ} \mathrm{C}$ for $10 \mathrm{~h}$, and then was cooled to room temperature, from which a white solid was precipitated. The solid was filtrated and washed with dichloromethane. The obtained solid was dried under reduced pressure to afford product $4(3.1 \mathrm{~g}, 84 \%) .{ }^{1} \mathrm{H}$ NMR (400 MHz, $\left.\mathrm{D}_{2} \mathrm{O}\right): \delta 4.87(\mathrm{~s}, 2 \mathrm{H}), 7.11-7.16(\mathrm{~m}, 4 \mathrm{H}), 7.30(\mathrm{~d}, J$ $=8.2 \mathrm{~Hz}, 2 \mathrm{H}), 7.40(\mathrm{~s}, 2 \mathrm{H}), 7.62-7.67(\mathrm{~m}, 4 \mathrm{H}), 7.89(\mathrm{~d}, J=7.9 \mathrm{~Hz}$, $2 \mathrm{H}), 8.19(\mathrm{~d}, J=4.4 \mathrm{~Hz}, 2 \mathrm{H}) .{ }^{13} \mathrm{C}$ NMR (101 MHz, DMSO- $\left.\mathrm{d}_{6}: \mathrm{H}_{2} \mathrm{O}=1: 3\right) \delta 69.4,111.8,118.1,118.1,122.2,124.7,126.8$, 128.2 , 135.9, 137.0, 138.2, 148.7, 148.8, 154.2, 155.2, 166.3, 172.2. HRMS (ESI): $m / z$ calcd for $\mathrm{C}_{29} \mathrm{H}_{19} \mathrm{~K}_{2} \mathrm{~N}_{4} \mathrm{O}_{5}: 581.0630[\mathrm{M}+$ $\mathrm{H}]^{+}$; found: 581.0625 .

Synthesis of oligomer-Ru(terpy)(pydic). A solution of $[\mathrm{Ru}(p$ cymene $\left.) \mathrm{Cl}_{2}\right]_{2}(0.70 \mathrm{~g}, 1.14 \mathrm{mmol})$ in DMF $(20 \mathrm{~mL})$ was added to a solution of $4(1.2 \mathrm{~g}, 2.07 \mathrm{mmol})$ in $\mathrm{DMF}-\mathrm{H}_{2} \mathrm{O}(1: 1,500 \mathrm{~mL})$ under argon atmosphere. After the mixture was stirred at $70{ }^{\circ} \mathrm{C}$ for $5 \mathrm{~h}$, a dark violet precipitate was formed. The precipitate collected by filtration was further purified by Soxhlet extraction for $10 \mathrm{~h}$ with ethanol and water to remove the impurities thoroughly and finally to give oligomer $\mathrm{Ru}$ (terpy)(pydic) (1.1 g, 88\%). Anal. Calc. for $\mathrm{C}_{116} \mathrm{H}_{72} \mathrm{~N}_{16} \mathrm{O}_{20} \mathrm{Ru}_{3} \mathrm{~K}_{2}: \mathrm{C}, 58.19 ; \mathrm{H}, 3.03 ; \mathrm{N}$, 9.37; found: C, 57.93; H, 2.92; N, 9.18.

Typical procedure for oxidation of alcohols. Substrate alcohol $(2.0 \mathrm{mmol})$, catalyst $(0.1 \mathrm{~mol} \%)$ and $n$-hexane $(2 \mathrm{~mL})$ were charged into a two necked flask. The mixture was heated to $50{ }^{\circ} \mathrm{C}$, then $70 \%$ TBHP $(6 \mathrm{mmol})$ was added slowly into the mixture in $0.5 \mathrm{~h}$ under stirring. The reaction mixture was further vigorously stirred at $50{ }^{\circ} \mathrm{C}$ to complete the reaction. After reaction, the product was isolated by silica gel column chromatography with mixture of $n$-hexane and ethyl acetate as eluent.

The procedure for the recyclation of the catalyst. 1-Phenylethanol $(60 \mathrm{mmol})$, catalyst $(0.06 \mathrm{mmol})$, and $n$-hexane $(60 \mathrm{~mL})$ were charged into a flask, successively. The mixture was heated to $50{ }^{\circ} \mathrm{C}$ under stirring. Then TBHP (70\% aqueous solution) (180 mmol) was slowly dropped into the mixture in $0.5 \mathrm{~h}$. After reaction $(9.5 \mathrm{~h})$, the catalyst was recovered by filtration and washed with ethanol, dried at $80{ }^{\circ} \mathrm{C}$ under vacuum, and then subjected to next run. In each subsequent cycle, the conversion of 1-phenylethanol was measured by GC after $9.5 \mathrm{~h}$.

\section{Results and discussion}

\section{Synthesis of oligomer-Ru(terpy)(pydic)}

As shown in Scheme 1, the ligand precursor called dimethyl 4-(4$\left(2,2^{\prime}: 6^{\prime}, 2^{\prime \prime}\right.$-terpyridin- $4^{\prime}$-yl)benzyloxy)pyridine-2,6-dicarboxylate (3) was smoothly obtained from 1 and 2 via Williamson reaction,

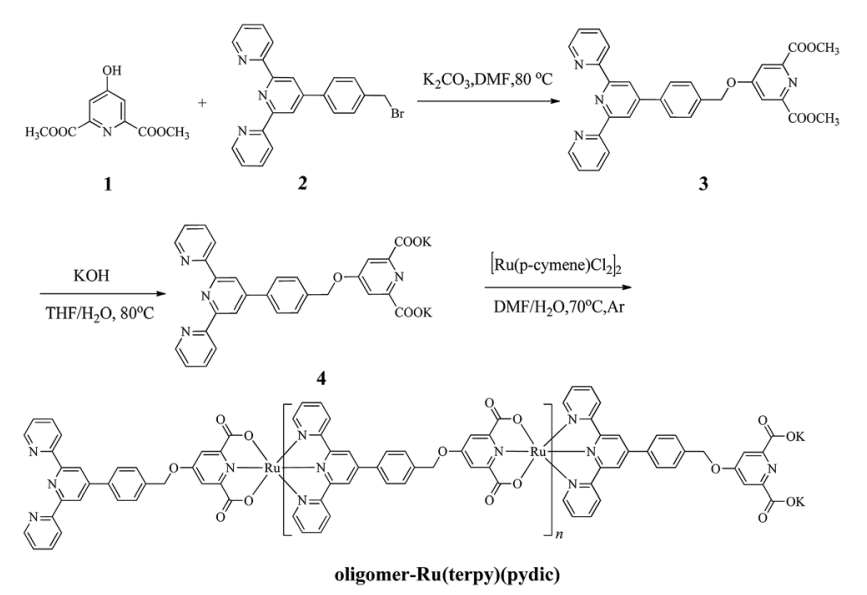

Scheme 1 Synthesis route for the oligomer-Ru(terpy)(pydic). 
which was confirmed by ${ }^{1} \mathrm{H}$ NMR and ${ }^{13} \mathrm{C}$ NMR. Hydrolysis of ligand precursor 3 in the presence of potassium hydroxide readily gave the ligand called potassium $4-\left(4-\left(2,2^{\prime}: 6^{\prime}, 2^{\prime \prime}\right.\right.$-terpyridin- $4^{\prime}$-yl $)$ benzyloxy)pyridine-2,6-dicarboxylate (4) which was thoroughly characterized by ${ }^{1} \mathrm{H}$ NMR, ${ }^{13} \mathrm{C}$ NMR, HR-MS, FT-IR and UV-vis spectroscopy.

The coordination of 4 with $\left[\mathrm{Ru}(p \text {-cymene }) \mathrm{Cl}_{2}\right]_{2}$ under the conditions similar to those for preparing $\mathrm{Ru}$ (terpy)(pydic) (p,51 $^{5,0}$ immediately afforded a dark violet solid product which was thoroughly characterized, and regarded as oligomer-Ru(terpy)(pydic).

\section{Characterization}

The synthesized solid product was firstly characterized by FT-IR. Fig. 1 shows the FT-IR spectra of ligand 4 (A) and oligomer$\mathrm{Ru}$ (terpy)(pydic) (B) in the range of $4000-400 \mathrm{~cm}^{-1}$. A broad band around $3445 \mathrm{~cm}^{-1}$ is observed, which is indicative the presence of potassium carboxylate in the structure. ${ }^{52}$ The strong band at $1594 \mathrm{~cm}^{-1}$ is from the $\mathrm{C}=\mathrm{N}$ and $\mathrm{C}=\mathrm{O}$ stretching vibration, ${ }^{53,54}$ and the bands at 1460,1404 , and $1333 \mathrm{~cm}^{-1}$ are associated with $\mathrm{C}-\mathrm{O}$ and $\mathrm{C}-\mathrm{N}$ stretching. ${ }^{55-58}$ Compared with that of the ligand, the spectrum of oligomer-Ru(terpy)(pydic) exhibits additional bands at $622 \mathrm{~cm}^{-1}$ and $468 \mathrm{~cm}^{-1}$ respectively, attributed to the $\mathrm{Ru}-\mathrm{O}$ and $\mathrm{Ru}-\mathrm{N}$ vibration. ${ }^{59,60}$ Besides, all the characteristic peaks in the IR spectrum of $\mathrm{Ru}$ (terpy)(pydic) based complexes ${ }^{61}$ can be found in that of oligomer-Ru(terpy)(pydic). To further confirm the formation of oligomer-Ru(terpy)(pydic), the Raman spectra of oligomer-Ru(terpy)(pydic) and its model complex $\mathrm{Ru}$ (terpy)(pydic) were taken and compared. As shown in Fig. 2, the spectrum (A) of oligomer-Ru(terpy)(pydic) is almost coincide with that (B) of Ru(terpy)(pydic). In the spectra, the bands at $1604 \mathrm{~cm}^{-1}$ and $1470 \mathrm{~cm}^{-1}$ can be ascribed to the stretching vibration of the $\mathrm{C}=\mathrm{N}$ from ligand pydic, and the bands at $1520 \mathrm{~cm}^{-1}$ and $1355 \mathrm{~cm}^{-1}$ are due to the stretching vibration of the $\mathrm{C}=\mathrm{N}$ from ligand terpy. The band at $650 \mathrm{~cm}^{-1}$ and the weak band at $455 \mathrm{~cm}^{-1}$ can be attributed to the $\mathrm{Ru}-\mathrm{O}$ and $\mathrm{Ru}-\mathrm{N}$ vibrations, respectively. Both the FT-IR and Raman spectra of oligomer$\mathrm{Ru}$ (terpy)(pydic) indicate that the obtained ruthenium complex has a Ru(terpy)(pydic) unit in its structure. Besides, the procedures for preparing oligomer-Ru(terpy)(pydic) are identical to those for the synthesis of $\mathrm{Ru}$ (terpy)(pydic). Therefore, we conclude that the

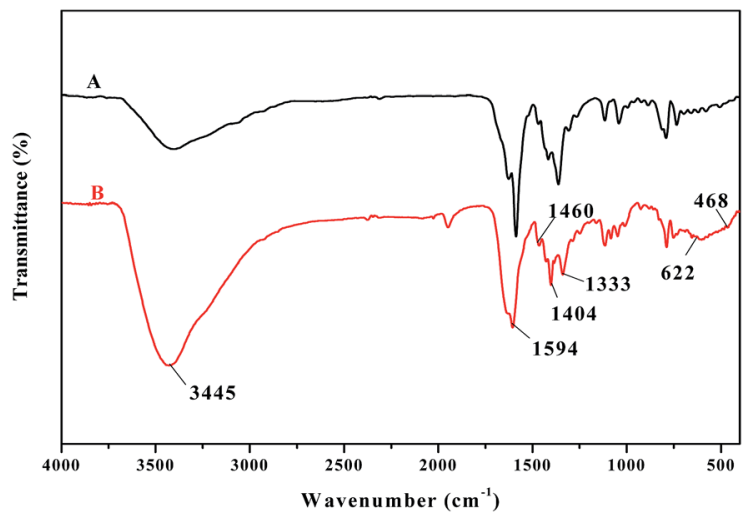

Fig. 1 FT-IR spectra of ligand 4 (A) and oligomer-Ru(terpy)(pydic) (B).

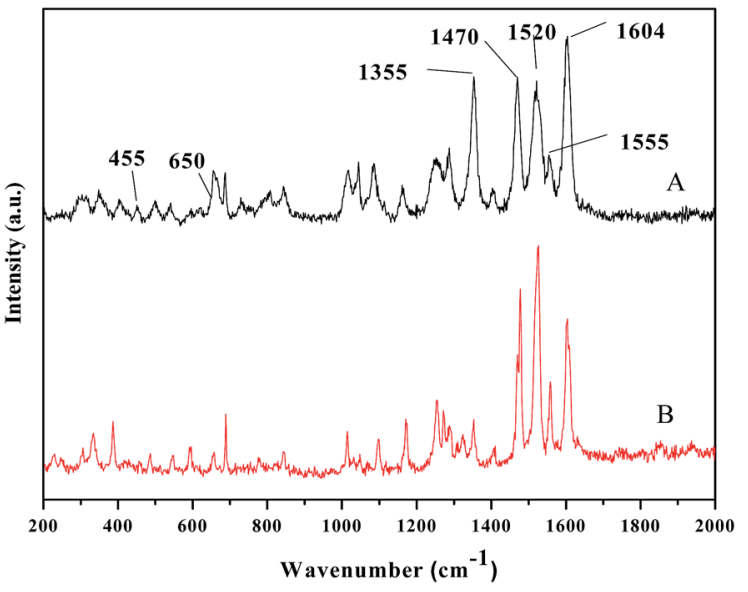

Fig. 2 Raman spectra of oligomer-Ru(terpy)(pydic) (A) and Ru(terpy)(pydic) (B).

oligomer-Ru(terpy)(pydic) has the structure as shown in Scheme 1, in which both a Ru(terpy)(pydic) unit and a potassium carboxylate moiety are involved. In the Ru(terpy)(pydic) unit, the terpy coordinates to central ruthenium atom via three pyridine nitrogens, and the pydic binds the metal center at the pyridine nitrogen and two carboxylate oxygens. The ligand terpy coordinates to $\mathrm{Ru}$ on one plane and the ligand pydic acts as a dianionic $O, N, O$-tridentate ligand on another plane. The two planes are nearly perpendicular to each other. ${ }^{62}$

ICP analysis revealed that the ruthenium content of the oligomer-Ru(terpy)(pydic) was $12.7 \mathrm{wt} \%$, from which it is calculated that the average polymerization degree of the obtained oligomer-Ru(terpy)(pydic) is about 4.0, and the $n$ in the structure shown in Scheme 1 is about 2.0. The elementary analysis results of $\mathrm{C}, \mathrm{H}$ and $\mathrm{N}$ are in agreement with the calculated ones based on the structure determined from ruthenium content.

The UV-vis DRS spectra for 4 (A) and oligomer-Ru(terpy)(pydic) (B) are showed in Fig. 3. In the spectrum of 4 , two bands maxima at 234 and $333 \mathrm{~nm}$ are derived from the $\pi-\pi^{*}$ transitions of phenyl group and pyridine ring, ${ }^{63,64}$ and no absorption band above $400 \mathrm{~nm}$ is found. Nevertheless, the spectrum of oligomer-

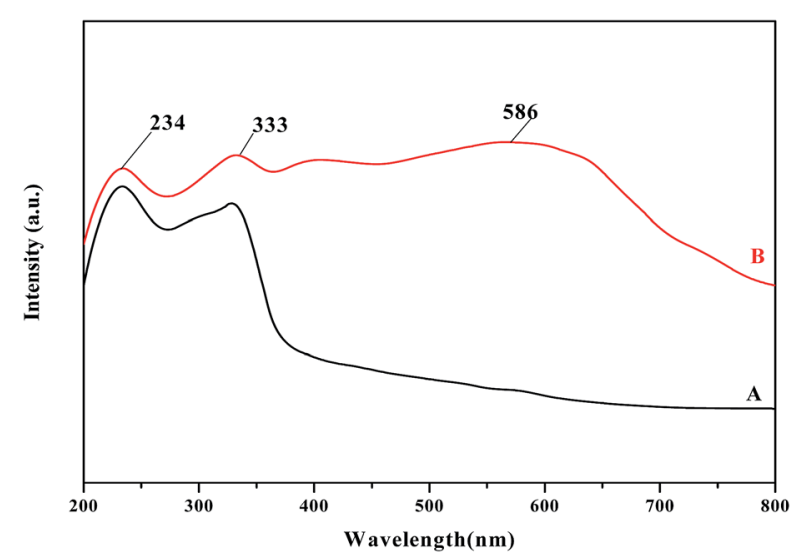

Fig. 3 UV-vis DRS spectra of 4 (A) and oligomer-Ru(terpy)(pydic) (B). 


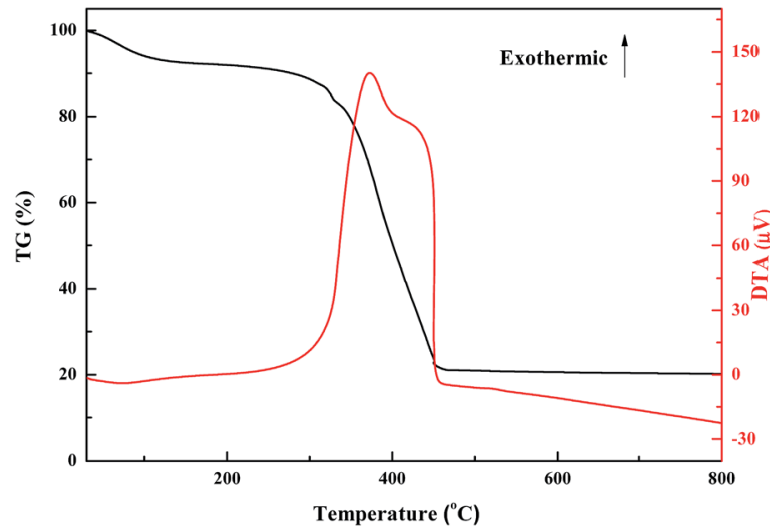

Fig. 4 TG and DTA curves of the thermal degradation of oligomerRu(terpy)(pydic).

$\mathrm{Ru}$ (terpy)(pydic) exhibits an additional broad band centered at $586 \mathrm{~nm}$, assigned to the metal-ligand charge transfer transition [MLCT, $\mathrm{d}(\mathrm{Ru}) \rightarrow \pi^{*}$ (pyridine)], which is a characteristic transition band in ruthenium polypyridine complexes. ${ }^{65,66}$

The thermal stability of oligomer-Ru(terpy)(pydic) was evaluated by thermal gravimetric analysis (Fig. 4). Two weight loss stages can be seen from the TG curve. The first endothermic stage occurring in the temperature range of $50-110{ }^{\circ} \mathrm{C}$ with a weight loss close to $7 \%$, is due to the desorption of physically adsorbed water. The second weight loss occurring in the range of 250 to $460{ }^{\circ} \mathrm{C}$ can be ascribed to the exothermic oxidative decomposition of oligomer-Ru(terpy)(pydic). These results proved that oligomer-Ru(terpy)(pydic) is thermally stable and could be used under $250{ }^{\circ} \mathrm{C}$.

Fig. 5(a) and (b) show the SEM images of the fresh and used samples of oligomer-Ru(terpy)(pydic), respectively. The images show that the morphologies of both the fresh and used samples composed of particles with irregular shapes and different
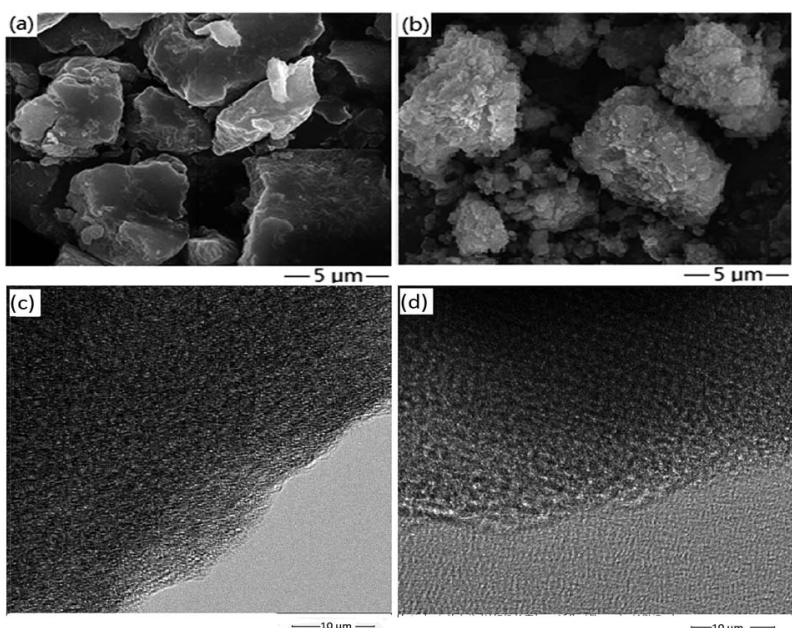

Fig. 5 (a) SEM image of the fresh oligomer-Ru(terpy)(pydic), (b) SEM image of the used oligomer-Ru(terpy)(pydic), (c) TEM image of the fresh oligomer-Ru(terpy)(pydic), (d) TEM image of the used oligomerRu(terpy)(pydic).

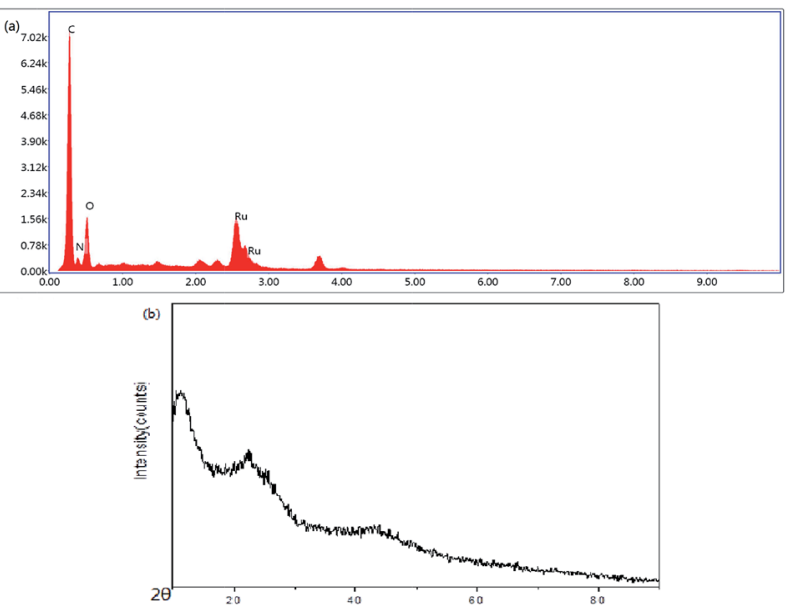

Fig. 6 (a) EDX analysis of oligomer-Ru(terpy)(pydic), (b) XRD analysis of oligomer-Ru(terpy)(pydic).

dimensions. The surfaces of the particles of the fresh sample are smooth, but the surfaces of the particles of the used one are rough. Besides, fragmentation of some particles is found in the used samples. These results indicated that some oligomer$\mathrm{Ru}$ (terpy)(pydic) molecules decomposed and peeled off from the surface during catalytic runs.

Fig. 5(c) and (d) exhibit the TEM of the fresh and used samples of oligomer-Ru(terpy)(pydic), respectively. As can be seen that the fresh sample has a porous structure composed of small particles, and no ruthenium aggregates are found in the structure. After catalytic run, the used sample shows big pores compared to the fresh one, indicating the peeling off of the surface oligomer-Ru(terpy)(pydic) molecules on the particles due to their decomposition during catalytic runs. No ruthenium aggregates are found, too. The TEM results are in accordance with those from SEM analysis.

The chemical composition of oligomer-Ru(terpy)(pydic) was characterized by energy dispersive spectroscopy. As shown in Fig. 6(a), the EDX spectrum exhibits peaks for $\mathrm{C}, \mathrm{N}, \mathrm{O}$ and $\mathrm{Ru}$, which are the component elements of oligomer Ru(terpy)(pydic). The EDX analysis of the fresh sample of oligomer$\mathrm{Ru}$ (terpy)(pydic) results showed the molar ratios for $\mathrm{N}$ : $\mathrm{O}$ to be about $1: 1.31$, which is well matched with the result calculated from the structure of oligomer Ru(terpy)(pydic). From EDX results the Ru content was calculated to be $13.0 \mathrm{wt} \%$, which is in agreement with the result from ICP analysis. Fig. 6(b) shows the $\mathrm{XRD}$ patterns of the sample of oligomer-Ru(terpy)(pydic) and no diffraction peaks representing ruthenium species are observed, which indicates that ruthenium does not exist in the form of inorganic particles in the sample.

The surface chemical composition and the oxidation state of ruthenium in oligomer-Ru(terpy)(pydic) were investigated by XPS analysis. The full-range XPS spectra (Fig. 7(a)) clearly showed characteristic peaks of $\mathrm{O}, \mathrm{N}$ and Ru elements. The narrows scan spectra of elements $\mathrm{C}$ 1s, Ru 3d and Ru 3p are shown in Fig. 7(b and c), respectively. The appearance of $\mathrm{Ru} 3 \mathrm{~d}_{5 / 2}(280.2 \mathrm{eV})$ and $\mathrm{Ru}$ $3 p_{3 / 2}(462.2 \mathrm{eV})$ peaks makes it evident that the ruthenium is present in $\mathrm{Ru}^{2+}$ state in the oligomer complex. ${ }^{67,68}$ 


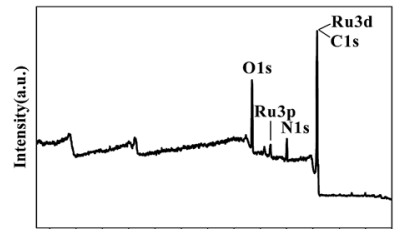

(a) Binding Energy $(\mathrm{eV})$

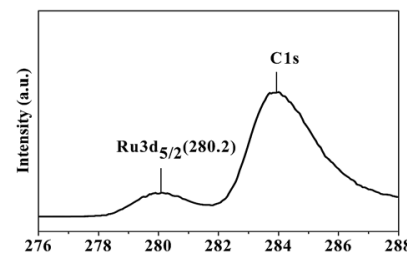

(b)

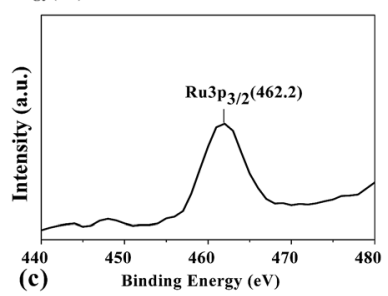

Fig. 7 XPS spectra of oligomer-Ru(terpy)(pydic): (a) wide view, (b) C 1s and Ru 3d, (c) Ru 3p spectra.

\section{Catalytic properties of oligomer-Ru(terpy)(pydic)}

The oligomer ruthenium complex was evaluated in the oxidation of secondary alcohols as a catalyst. Initially, several oxidants including molecular oxygen, $\mathrm{H}_{2} \mathrm{O}_{2}$ and TBHP were tested in the reaction with 1-phenylethanol as a model substrate in cyclohexane (see Table $\mathrm{S} 1$ in the $\mathrm{ESI} \dagger$ ). No reaction was observed with molecular oxygen as oxidant. In the case of $\mathrm{H}_{2} \mathrm{O}_{2}$ as oxidant, the yield of the target product acetophenone was only $32 \%$ for $16 \mathrm{~h}$, which is different from that under the catalysis of $\mathrm{Ru}$ (terpy)(pydic) in literature. ${ }^{25}$ In that case, $\mathrm{H}_{2} \mathrm{O}_{2}$ showed good performance in the oxidation of secondary alcohols to the corresponding ketones. Under the same reaction conditions, the yield of acetophenone reached up to $96 \%$ with TBHP as oxidant. Next, the solvent was screened. In viewpoint of the yield of acetophenone, $n$-hexane was the best one among the screened solvents (see Table S1 in the ESI $\dagger$ ). After the determination of oxidant and solvent, the parameters such as the loadings of TBHP and catalyst, and the reaction temperature were optimized thoroughly (see Tables S2-S4 in the ESI $\dagger$ ). Based on the experimental results, the optimal reaction conditions for the oxidation of alcohols were obtained, which are alcohol substrate $2 \mathrm{mmol}$, catalyst $0.1 \mathrm{~mol} \%$, molar ratio of TBHP to substrate $3: 1$, reaction temperature $50{ }^{\circ} \mathrm{C}$, and $n$-hexane $2 \mathrm{~mL}$.

The substrate tolerance of the catalytic oxidation system was then investigated under the optimal reaction conditions. The results are compiled in Table 1 . As shown in Table 1, the catalytic oxidation system has good substrate tolerance. Various secondary alcohols including benzylic, linear and cyclic aliphatic ones were oxidized to their corresponding ketones. For the benzylic alcohols, the ones with whether an electrondonating or an electron-withdrawing group were almost oxidized to the corresponding ketones quantitatively, but the reaction time to finish the reaction was different (Table 1, entries 1-14). The substrate with an electron-withdrawing substituent para to the hydroxyalkyl group had high reactivity compared to the one with an electron-donating substituent, and the stronger the electron-withdrawing capacity of the substituent is, the shorter time is required to finish the reaction (Table
1, entries 2, 3, 6-8). The ortho-substituted substrates reacted slowly compared to the para- or meta-isomer due to the steric hindrance of between the substituent and the hydroxyalkyl group. For examples, long reaction time and high TBHP loading were required in the oxidation of 1-(2-chlorophenyl) ethanol and 1-(2-methoxylphenyl)ethanol compared to that of 1-(4chlorophenyl) ethanol and 1-(4-methoxylphenyl) ethanol (Table 1 , entries $3,5,8,9)$. In the oxidation of 2-pentanol and cyclohexanol which are the representatives of linear and cyclic secondary aliphatic alcohols, excellent yields were also obtained though long reaction time and high TBHP loading were needed (Table 1, entries 15, 18, 19). However, in the oxidation of 2isopropyl-5-methylcyclohexanol poor result was received due to iso-propyl ortho to the hydroxyl leading to big steric hindrance in the structure (Table 1, entries 16,17). The catalytic oxidation system was also evaluated in the oxidation of some primary benzylic alcohols. In all the cases the primary alcohols were over oxidized to the corresponding carboxylic acids (Table 1, entries 20-23). Because the aldehydes from alcohol were more easily oxidized than alcohols, more TBHP was consumed in the reaction of aldehydes to acids leading to low conversion of alcohols. Meanwhile, it is difficult to control the selectivity either to aldehydes or carboxylic acids. High TBHP loading was in favor of the reaction to the acid completely. For instance, both the conversion of alcohol and the yield of acid can approach $100 \%$ at $10 \mathrm{mmol}$ TBHP loading (Table 1, entry 24). Finally, 4-tert-butoxyphenethyl alcohol and 3-phenyl-1-propanol as representatives of the primary aliphatic alcohols were tested in the oxidation by the catalytic oxidative system. However, the expected products 4-tert-butoxyphenylacetic acid and 3-phenylpropionic acid were not obtained. Interestingly, the former was transformed to 4-tert-butoxybenzoic acid, and the latter was oxidized to 3-hydroxy-1-phenylpropan-1-one, respectively (Table 1, entries 25-26). Both of the products were derived from the oxidation of benzylic methylene of substrates.

The results in Table 1 indicate that this catalytic system is more suitable for the selective oxidation of the secondary alcohols to the corresponding ketones, which is complementary with the copper/TEMPO catalyzed aerobic oxidation of the primary alcohols to aldehydes. ${ }^{\mathbf{1 4 , 6 9 , 7 0}}$ Generally, the copper/ TEMPO based aerobic oxidation systems are not efficient in the oxidation of the secondary alcohols to ketones. This oligomer-Ru(terpy)(pydic) is only efficient in the case of TBHP as oxidant, however, the homogeneous ruthenium complexes with a similar structure unit to it are generally active in the oxidation of alcohols with $\mathrm{H}_{2} \mathrm{O}_{2}{ }^{24,25}$ It is obvious that $\mathrm{H}_{2} \mathrm{O}_{2}$ is more environmentally friendly than TBHP.

Additional experiments were conducted to evaluate the heterogeneity of the reaction catalyzed by oligomer$\mathrm{Ru}$ (terpy)(pydic) with 1-phenylethanol as the substrate. First, oligomer-Ru(terpy)(pydic) (0.1 mol\%) was suspended in the mixture of $n$-hexane $(2 \mathrm{~mL})$ and 1-phenylethanol $(2 \mathrm{mmol})$ under stirring at $50{ }^{\circ} \mathrm{C}$ for eight hours being the same time in the oxidation of 1-phenylethanol by TBHP. The solid catalyst was removed from the mixture by hot filtration. To the filtrate was added TBHP (6 mmol) in dropwise and the mixture was stirred at $50{ }^{\circ} \mathrm{C}$ for eight hours. Only $24 \%$ of the conversion of 1 - 
Table 1 Oxidation of various alcohols with TBHP catalyzed by oligomer-Ru(terpy)(pydic) ${ }^{a}$

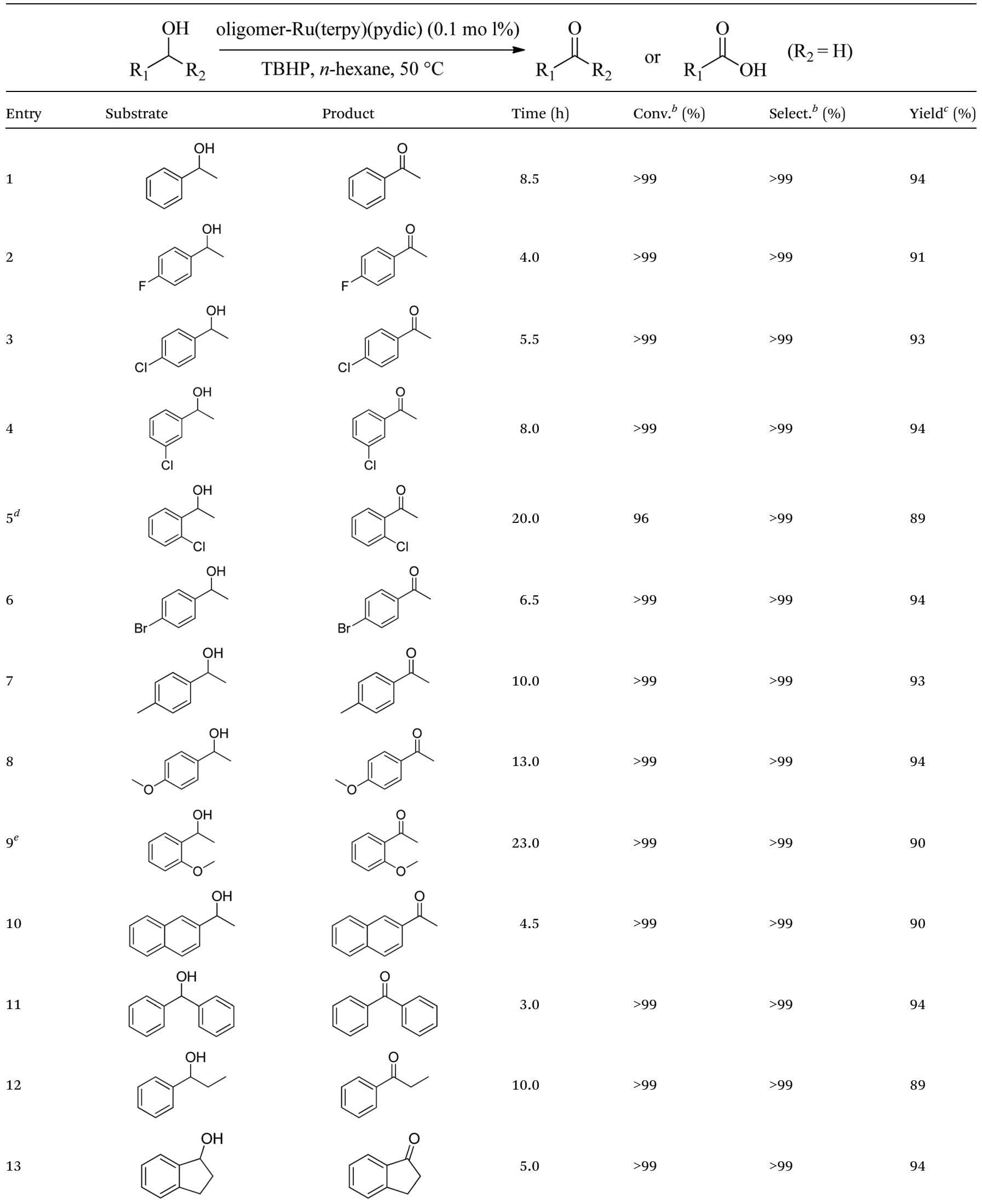


Table 1 (Contd.)

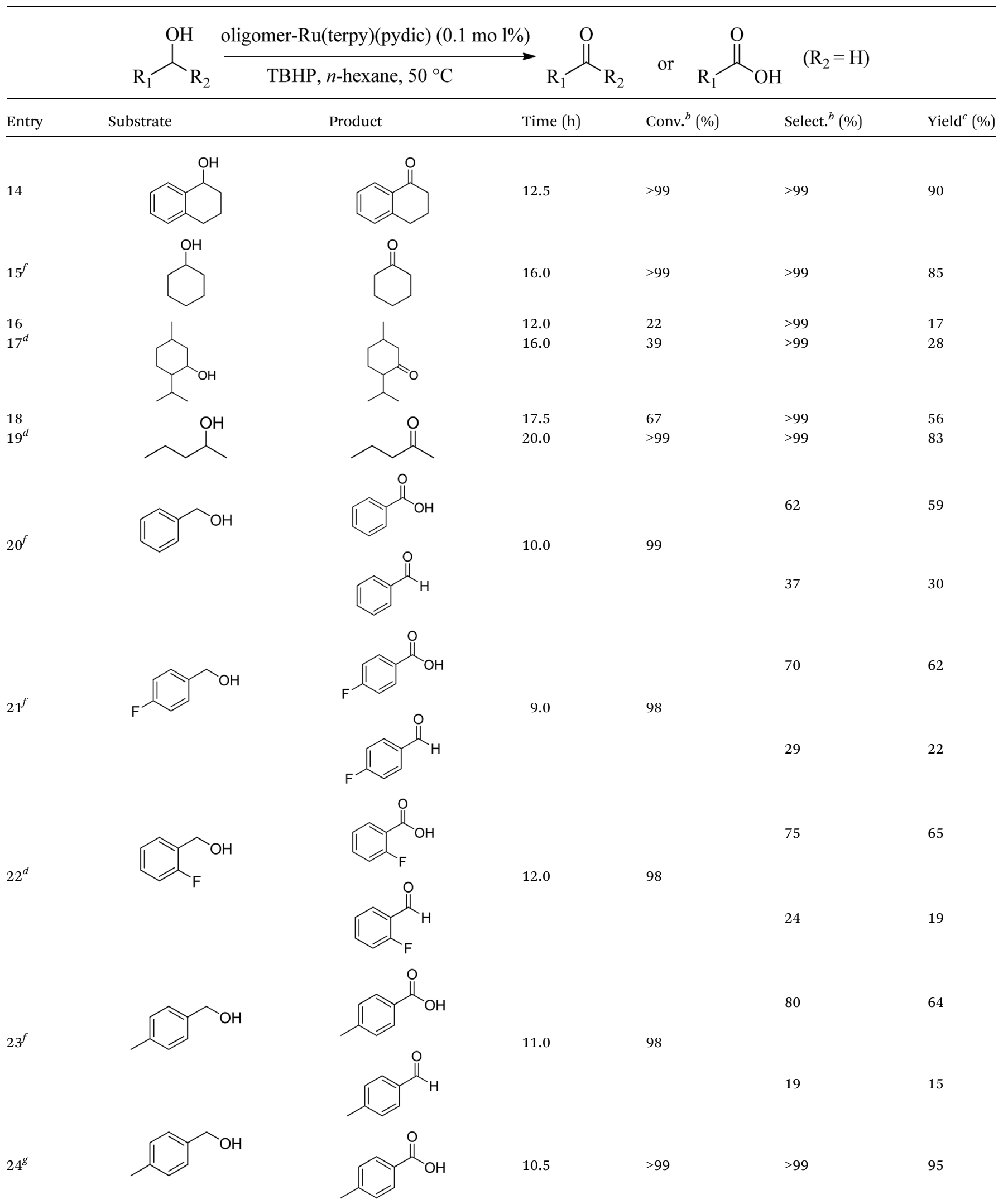


Table 1 (Contd.)

Entry

\begin{abstract}
${ }^{a}$ Reaction condition: 1-phenylethanol $(2 \mathrm{mmol})$, oxidant $(6 \mathrm{mmol})$, catalyst $(0.1 \mathrm{~mol} \%), n$-hexane $(2 \mathrm{~mL})$, reaction temperature $50{ }^{\circ} \mathrm{C} .{ }^{b}$ Conversions and selectivity were determined by GC (area normalization method). ${ }^{c}$ Isolated yields, the product was purified by column chromatography over silica gel (eluent: $n$-hexane/ethyl acetate), all products were determined by ${ }^{1} \mathrm{H}$ NMR. ${ }^{d}$ Oxidant: 8 mmol. ${ }^{e}$ Oxidant: 7 mmol. ${ }^{f}$ Oxidant: 7.5 mmol. ${ }^{g}$ Oxidant: $10 \mathrm{mmol}$.
\end{abstract}

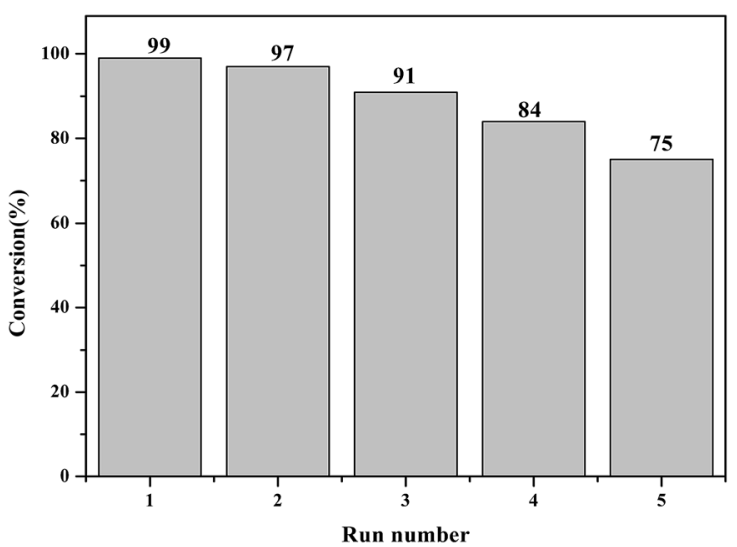

Fig. 8 Recycling of oligomer-Ru(terpy)(pydic) in the oxidation of 1phenylethanol. Reaction condition: 1-phenylethanol $(60 \mathrm{mmol})$, catalyst $(0.06 \mathrm{mmol})$, oxidant $(180 \mathrm{mmol}), n$-hexane $(60 \mathrm{~mL})$, temperature $50{ }^{\circ} \mathrm{C}$, reaction time $9.5 \mathrm{~h}$.

phenylethanol was observed. In another experiment, to the mixture of $n$-hexane $(2 \mathrm{~mL})$ and 1-phenylethanol $(2 \mathrm{mmol})$ without catalyst was added TBHP (6 mmol) in dropwise under stirring at $50{ }^{\circ} \mathrm{C}$ for eight hours, too. 1-Phenylethanol was converted to acetophenone in a conversion of $22 \%$ in the absence of catalyst, which is close to the above result $(24 \%)$ of the oxidation of the hot filtrate. The results indicated that oligomer-Ru(terpy)(pydic) is almost insoluble in $n$-hexane, and oligomer-Ru(terpy)(pydic) is belong to a heterogeneous catalyst.

\section{The recyclability of the catalyst}

Further experiments were performed to examine the reusability of oligomer-Ru(terpy)(pydic) with 1-phenylethanol as a model substrate. The procedures have been given in the experimental section. The recycle test results are displayed in Fig. 8. As shown in the figure, the conversion decreased gradually with recycle times but still reached $84 \%$ in the fourth run. It can be concluded that the catalyst could be recycled at least four cycles without a large reduction in its activity and selectivity. The oligomer-Ru(terpy)(pydic) showed better performance than the organic polymer supported ruthenium complexes but poorer performance than the inorganic immobilized ruthenium complexes..$^{27,40-43}$ The ruthenium content of the samples of the fresh catalyst and recovered one after the fifth run was determined by ICP to be $12.7 \mathrm{wt} \%$ and $10.5 \mathrm{wt} \%$, respectively. The ruthenium loss is approximately $17.3 \%$. This result in combination with the SEM images of the fresh and used samples indicates that the decrease in activity of the oligomer ruthenium complex was mainly due to its light decomposition during catalytic run.

\section{Conclusions}

A ligand with both terpyridine and pyridine-2,6-dicarboxylate functional groups were designed and synthesized. The ligand can coordinate with $\mathrm{Ru}^{2+}$ to give an oligomer ruthenium complex with an average polymerization degree of 4.0 named as oligomer-Ru(terpy)(pydic). This oligomer ruthenium complex as a heterogeneous catalyst showed high activity and selectivity in the oxidation of various secondary alcohols to the corresponding ketones with TBHP as oxidant. The oligomer ruthenium complex can be easily separated and recycled several times. The gradual deactivation of this complex is due to its slight decomposition during the catalytic run.

\section{Conflicts of interest}

There are no conflicts to declare. 


\section{Acknowledgements}

This work was financially supported by the National Natural Science Foundation of China (No. 21476057 and 21276061), the Program for the Top Young Innovative Talents of Hebei Province, China (Grant No. BJ2017010), Tianjin Municipal Natural Science Foundation (Grant No. 15JCYBJC21000), Foundation of Education Bureau of Hebei Province (Grant No. QN2016152).

\section{Notes and references}

1 M. Hudlicky, Oxidations in Organic Chemistry, ACS, Washington, DC, 1990.

2 D. H. Pybus and C. S. Sell, The Chemistry of Fragrances, RSC Paperbacks, Cambridge, 1999.

3 R. A. Sheldon, I. W. C. E. Arends and A. Dijksman, Catal. Today, 2000, 57, 157-166.

4 T. Mallat and A. Baiker, Chem. Rev., 2004, 104, 3037-3058.

5 J. March, Advanced organic chemistry: reactions, mechanisms, and structure, Wiley, New York, 1992.

6 R. C. Larock, Comprehensive organic transformations: a guide to functional group preparations, Wiley-VCH, New York, 1999.

7 G. Tojo and M. Fernández, Oxidations of alcohols to aldehydes and ketones: a guide to current common practice, Springer, Boston, MA, 2006.

8 R. A. Sheldon and J. K. Kochi, Metal-catalyzed oxidations of organic compounds, Academic Press, New York, 1981.

9 D. B. Dess and J. C. Martin, J. Org. Chem., 1983, 48, 41554156.

10 D. B. Dess and J. C. Martin, J. Am. Chem. Soc., 1991, 113, 7277-7287.

11 R. Alvarez, B. Iglesias, S. López and A. R. Lera, Tetrahedron Lett., 1998, 39, 5659-5662.

12 J. D. More and N. S. Finney, Org. Lett., 2002, 4, 3001-3003.

13 M. Frigerio, M. Santagostino, S. Sputore and G. Palmisano, J. Org. Chem., 1995, 60, 7272-7276.

14 P. Gamez, I. W. C. E. Arends, J. Reedijk and R. A. Sheldon, Chem. Commun., 2003, 19, 2414-2415.

15 P. Gamez, I. W. C. E. Arends, R. A. Sheldon and J. Reedijk, Adv. Synth. Catal., 2004, 346, 805-811.

16 M. N. Kopylovich, K. T. Mahmudov, M. Haukka, P. J. Figiel, A. Mizar, J. A. L. Silva and A. J. L. Pombeiro, Eur. J. Inorg. Chem., 2011, 27, 4175-4181.

17 W. Yin, C. Chu, Q. Lu, J. Tao, X. Liang and R. Liu, Adv. Synth. Catal., 2010, 352, 113-118.

18 M. R. Maurya, N. Saini and F. Avecilla, Inorg. Chim. Acta, 2015, 438, 168-178.

19 G. D. Yadav and A. R. Yadav, J. Mol. Catal. A: Chem., 2013, 380, 70-77.

20 G. Sarmah, S. K. Bharadwaj, A. Dewan, A. Gogoi and U. Bora, Tetrahedron Lett., 2014, 55, 5029-5032.

21 J. K. Mobley and M. Crocker, RSC Adv., 2015, 5, 65780-65797.

22 M. Lenze and E. B. Bauer, Chem. Commun., 2013, 49, 58895891.

23 B. M. Peterson, M. E. Herried, R. L. Neve and R. W. McGaff, Dalton Trans., 2014, 43, 17899-17903.
24 F. Shi, M. K. Tse and M. Beller, Chem.-Asian J., 2007, 2, 411415.

25 X. T. Zhou, H. B. Ji and S. G. Liu, Tetrahedron Lett., 2013, 54, 3882-3885.

26 Y. Zhang, L. Liu, X. Cao and J. Zhao, Polyhedron, 2016, 105, 170-177.

27 W. Cheung, W. Yu, W. Yip, N. Zhu and C. Che, J. Org. Chem., 2002, 67, 7716-7723.

28 S. Muthusamy, N. Kumarswamyreddy, V. Kesavan and S. Chandrasekaran, Tetrahedron Lett., 2016, 57, 5551-5559.

29 M. Yamaguchi, H. Kousaka, S. Izawa, Y. Ichii, T. Kumano, D. Masui and T. Yamagishi, Inorg. Chem., 2006, 45, 83428354.

30 E. Vanover, Y. Huang, L. Xu, M. Newcomb and R. Zhang, Org. Lett., 2010, 12, 2246-2249.

31 F. Weisser, H. Stevens, J. Klein, M. V. Meer, S. Hohloch and B. Sarkar, Chem.-Eur. J., 2015, 21, 8926-8938.

32 T. Kojima, K. Nakayama, K. Ikemura, T. Ogura and S. Fukuzumi, J. Am. Chem. Soc., 2011, 133, 11692-11700.

33 T. Ishizuka, S. Ohzu and T. Kojima, Synlett, 2014, 25, 16671679.

34 K. Jitsukawa, Y. Oka, H. Einaga and H. Masuda, Tetrahedron Lett., 2001, 42, 3467-3469.

35 Y. Hirai, T. Kojima, Y. Mizutani, Y. Shiota, K. Yoshizawa and S. Fukuzumi, Angew. Chem., Int. Ed., 2008, 47, 5772-5776.

36 A. Taketoshi, X. N. Beh, J. Kuwabara, T. Koizumi and T. Kanbara, Tetrahedron Lett., 2012, 53(28), 3573-3576.

37 S. Manzini, C. A. Urbina-Blanco and S. P. Nolan, Organometallics, 2013, 32(2), 660-664.

38 S.-S. Wang, J. Zhang, C.-L. Zhou, G. Vo-Thanh and Y. Liu, Catal. Commun., 2012, 28, 152-154.

39 H. Mizoguchi, T. Uchida and T. Katsuki, Angew. Chem., Int. Ed., 2014, 53(12), 3178-3182.

40 H.-Y. Shen, S.-C. Zhou, M.-H. Wei and H.-X. Zong, React. Funct. Polym., 2006, 66(8), 827-831.

41 T. Matsumoto, M. Ueno, N. Wang and S. Kobayashi, Chem.Asian J., 2008, 3(2), 239-243.

42 S. Ganesamoorthy, M. M. Tamizh, K. Shanmugasundaram and R. Karvembu, Tetrahedron Lett., 2013, 54(51), 7035-7039.

43 Y. Zhang, X. Sun, H. Zhang and J. Zhao, Appl. Organomet. Chem., 2016, 30, 645-652.

44 Y. Zhang, R. Chu, H. Zhang and J. Zhao, Transition Met. Chem., 2017, 42, 105-116.

45 G. Howáth, C. Rusa, Z. Köntös, J. Gerencsér and P. Huszthy, Synth. Commun., 1999, 29, 3719-3731.

46 X. Yin and M. Tan, Zhongguo Xitu Xuebao, 2002, 20, 240-243.

47 X. Zhang, D. Huang, Y. Chen and R. H. Holm, Inorg. Chem., 2012, 51, 11017-11029.

48 J. Xu, R. Wang, Y. Li, Z. Gao, R. Yao, S. Wang and B. Wu, Eur. J. Inorg. Chem., 2012, 3349-3360.

49 B. Tang, F. Yu, P. Li, L. Tong, X. Duan, T. Xie and X. Wang, J. Am. Chem. Soc., 2009, 131, 3016-3023.

50 H. Nishiyama, T. Shimada, H. Itoh, H. Sugiyama and Y. Motoyama, Chem. Commun., 1997, 1863-1864.

51 M. Tse, S. Bhor, M. Klawonn, G. Anilkumar, H. Jiao, C. Dçbler, A. Spannenberg, W. Maegerlein, H. Hugl and M. Beller, Chem.-Eur. J., 2006, 12, 1855-1874. 
52 M. Gasgnier, J. Mater. Sci. Lett., 2001, 20, 1259-1262.

53 M. Karnan, V. Balachandran, M. Murugan and M. K. Murali, Spectrochim. Acta, Part A, 2014, 130, 143-151.

54 L. H. Abdel-Rahman, A. M. Abu-Dief, M. Ismael, M. A. A. Mohamed and N. A. Hashem, J. Mol. Struct., 2016, 1103, 232-244.

55 A. Patel and S. Singh, Microporous Mesoporous Mater., 2014, 195, 240-249.

56 A. I. Vogel, A text book of quantitative inorganic analysis, Longmans, London, 1994.

57 Y. F. Xie, H. Zhu, H. T. Shi, A. Q. Jia and Q. F. Zhang, Inorg. Chim. Acta, 2015, 428, 147-153.

58 T. S. Kamatchi, N. Chitrapriya, H. Lee, C. F. Fronczek, F. R. Fronczek and K. Natarajan, Dalton Trans., 2012, 41, 2066-2077.

59 Z. W. Yang, Q. X. Kang, F. Quan and Z. Q. Lei, J. Mol. Catal. A: Chem., 2007, 261, 190-195.

60 L. H. Abdel-Rahman, A. M. Abu-Dief, M. S. S. Adam and S. K. Hamdan, Catal. Lett., 2016, 146, 1373-1396.

61 K. Isozaki, T. Yokoi, R. Yoshida, K. Ogata, D. Hashizume, N. Yasuda, K. Sadakane, H. Takaya and M. Nakamura, Chem.-Asian J., 2016, 11, 1076-1091.
62 S. M. Couchman, J. M. Dominguez-Vera, J. C. Jeffery, C. A. McKee, S. Nevitt, M. Pohlman, C. M. White and M. D. Ward, Polyhedron, 1998, 17, 3541-3550.

63 J. Wang, C. Wang, K. E. deKrafft and W. Lin, ACS Catal., 2012, 2, 417-424.

64 G. Swiderski, M. Kalinowska, J. Malejko and W. Lewandowski, Vib. Spectrosc., 2016, 87, 81-87.

65 M. M. Tamizh, K. Mereiter, K. Kirchner and R. Karvembu, J. Organomet. Chem., 2012, 700, 194-198.

66 S. C. Yu, S. Hou and W. K. Chan, Macromolecules, 1999, 32, 5251-5256.

67 J. F. Moulder, W. F. Stickle, P. E. Sobol and K. D. Bomben, Handbook of X-ray Photoelectron Spectroscopy, Physical Electronics Division, Perkin-Elmer, Eden Prairie, MN, 1992.

68 J. Y. Shen, A. Adnot and S. Kaliaguine, Appl. Surf. Sci., 1991, 51, 47-60.

69 I. E. Markó, A. Gautier, R. Dumeunier, K. Doda, F. Philippart, S. M. Brown and C. J. Urch, Angew. Chem., Int. Ed., 2004, 43, 1588-1591.

70 J. M. Hoover and S. S. Stahl, J. Am. Chem. Soc., 2011, 133, 16901-16910. 